\title{
An origin for short $\gamma$-ray bursts unassociated with current star formation
}

S. D. Barthelmy ${ }^{1}$, G. Chincarini ${ }^{2,3}$, D. N. Burrows ${ }^{4}$, N. Gehrels ${ }^{1}$, S. Covino $^{2}$, A, Moretti $^{2}$, P. Romano ${ }^{2}, \mathrm{P}$

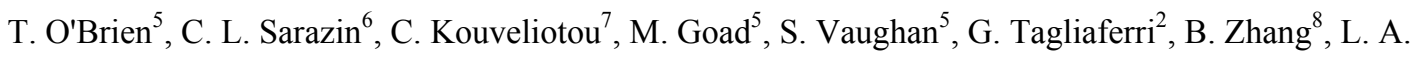
Antonelli $^{9}$, S. Campana ${ }^{2}$, J. R. Cummings ${ }^{1,10}$, P. D'Avanzo ${ }^{2,11}$, M. B. Davies ${ }^{12}$, P. Giommi ${ }^{13}$, D. Grupe ${ }^{4}$, Y. Kaneko ${ }^{14}$, J. A. Kennea ${ }^{4}$, A. King ${ }^{5}$, S. Kobayashi ${ }^{4}$, A. Melandri ${ }^{9}$, P. Meszaros ${ }^{4,15}$, J. A. Nousek ${ }^{4}$, S. Patel $^{14}$, T. Sakamoto ${ }^{1,10}$ \& R. A. M. J. Wijers ${ }^{16}$

${ }^{I}$ NASA/Goddard Space Flight Center Greenbelt, Maryland 20771, USA. ${ }^{2}$ INAF-Osservatorio Astronomico di Brera, Via Bianchi 46, I-23807 Merate, Italy. ${ }^{3}$ Universita degli studi di Milano Bicocca, Piazza delle Scienze 3, I-20126 Milano, Italy. ${ }^{4}$ Department of Astronomy and Astrophysics, Penn State University, University Park, Pennsylvania 16802, USA. ${ }^{5}$ Department of Physics and Astronomy, University of Leicester, Leicester LE1 7RH, UK. ${ }^{6}$ Department of Astronomy, University of Virginia, Charlottesville, Virginia 22903-0818, USA. ${ }^{7}$ NASA/Marshall Space Flight Center, NSSTC, XD-12, 320 Sparkman Drive, Huntsville, Alabama 35805, USA. ${ }^{8}$ Department of Physics, University of Nevada, Las Vegas, Las Vegas, Nevada 89154-4002, USA. ${ }^{9}$ Osservatorio Astronomico di Roma, Via di Frascati, 33 00040 Monte Porzio Catone, Italy. ${ }^{10}$ National Research Council, 2101 Constitution Avenue NW, Washington, DC 20418, USA. ${ }^{11}$ Universita degli Studi dell'Insubria, Dipartimento di Fisica e Matematica, via Valleggio 11, 22100 Como, Italy. ${ }^{12}$ Lund Observatory, Box 43, SE-221 00 Lund, Sweden. ${ }^{13}$ ASI Science Data Center, Via Galileo Galilei, I-00044 Frascati, Italy. ${ }^{14}$ Universities Space Research Association, NSSTC, XD-12, 320 Sparkman Drive, Huntsville, Alabama 35805, USA. ${ }^{15}$ Department of Physics, Penn State University, University Park, Pennsylvania 16802, USA. ${ }^{16}$ Astronomical Institute 'Anton Pannekoek', University of Amsterdam, Kruislaan 403, 1098 SJ Amsterdam, The Netherlands.

Two short ( $<2$ s) $\gamma$-ray bursts (GRBs) have recently been localized ${ }^{1-4}$ and fading afterglow counterparts detected ${ }^{2-4}$. The combination of these two results left unclear the nature of the host galaxies of the bursts, because one was a starforming dwarf, while the other was probably an elliptical galaxy. Here we report the X-ray localization of a short burst (GRB 050724) with unusual $\gamma$-ray and X-ray properties. The $\mathrm{X}$-ray afterglow lies off the centre of an elliptical galaxy at a redshift of $z=0.258$ (ref. 5), coincident with the position determined by groundbased optical and radio observations ${ }^{6-8}$. The low level of star formation typical for elliptical galaxies makes it unlikely that the burst originated in a supernova explosion. A supernova origin was also ruled out for GRB 050709 (ref. 3), even though that burst took place in a galaxy with current star formation. The isotropic energy for the short bursts is 2-3 orders of magnitude lower than that for the long 


\section{bursts. Our results therefore suggest that an alternative source of bursts- the coalescence of binary systems of neutron stars or a neutron star-black hole pair-are the progenitors of short bursts.}

On 2005 July 24 at 12:34:09.32 UT (Universal Time), the BAT instrument on the Swift observatory ${ }^{9}$ triggered and located GRB 050724 (ref. 6). The lightcurve for this event (Fig. 1) is somewhat different from the typical short GRB population as originally defined by the Burst and Transient Source Experiment (BATSE) ${ }^{10}$. Similar to GRB 050709 (ref. 11), the event consists of two parts that are spectrally very different. The main portion has a duration of $3.0 \pm 1.0 \mathrm{~s}$ and is dominated by a hard spike lasting only $0.25 \mathrm{~s}$. Following the main peak at time $T$, there is a second smaller peak at $T+1.1 \mathrm{~s}$ plus long-lasting faint emission out to $T+140 \mathrm{~s}$, which is all in the $15-25 \mathrm{keV}$ band. The faint tail images to the same location on the sky as the initial hard spike and is therefore definitely part of the initial event. The fluence in the faint tail is only $10 \%$ of that in the main peak.

The hardness ratio (50-100 keV to $25-50 \mathrm{keV}$ ) of the first peak is $0.91 \pm 0.12$, which is on the low end of the distribution for short GRBs as detected with the BATSE instrument. The hardness ratio is $0.55 \pm 0.23$ for the second peak at $T+1.1 \mathrm{~s}$ and $0.31 \pm 0.10$ for the long-lasting emission out to $T+140 \mathrm{~s}$. To determine whether this GRB would indeed have been classified as short in the BATSE sample, we performed a series of 100 simulations each, using the BATSE large-area detector response matrices for three different angles of incidence, for all main constituents of GRB 050724 . We found that in its typical trigger energy range (50-300 keV), BATSE would have triggered on the main hard peak and would definitely have detected the second pulse at $T+1.1 \mathrm{~s}$; however, the softer pulse starting at $T+30 \mathrm{~s}$ would have been detected at best at the $0.3 \sigma$ level and, thus, it would have not been accounted as part of the GRB. As a final test we passed the entire BAT lightcurve collected between 50-350 keV through the BATSE $T_{90}$ algorithm, obtaining $T_{90}=1.31 \pm 0.53 \mathrm{~s}$, entirely consistent with the short GRB population (but does not rule out a long-burst origin). Therefore, the long-lasting emission of this burst is probably a newly detected component of short bursts detected by BAT with its $15 \mathrm{keV}$ threshold and not seen by BATSE with its $>25 \mathrm{keV}$ threshold (although possibly hinted at in the sum of multiple short BATSE GRBs ${ }^{12,13}$ ).

The Swift X-Ray Telescope (XRT) began taking data $74 \mathrm{~s}$ after the BAT trigger $^{6}$, and located a bright, fading X-ray source, identified as the X-ray afterglow ${ }^{14,15}$. Ground-based observatories detected radio $^{7}$ and optical ${ }^{16,17}$ afterglows within the XRT error circle and we obtained a high-precision X-ray position ${ }^{8}$ with our Chandra X-ray Observatory target of opportunity observation. The positions determined by the various 
instruments are given in Table 1 and shown in Fig. 2. The radio, optical, and X-ray positions are all coincident.

The fading afterglow of GRB 050724 was located ${ }^{18}$ approximately 1" south of the centre of (but within) a bright elliptical galaxy at a redshift of $z=0.258$ (ref. 5). The projected offset from the centre of the galaxy corresponds to $\sim 4 \mathrm{kpc}$ (see Fig. 2). The host galaxy has an apparent magnitude of $K=15.3$ within a 3" radius aperture ${ }^{17}$, which corresponds to a luminosity of $2 \times 10^{11} L_{\mathrm{O}} \approx 1.7 L^{*}$ (where $L_{\mathrm{O}}$ and $L^{*}$ are the luminosities of the Sun and a typical galaxy, respectively). The total luminosity is probably slightly larger. Galaxies this luminous are relatively uncommon, with a comoving number density ${ }^{19}$ of only $3 \times 10^{-4} \mathrm{Mpc}^{-3}$. The probability that a GRB would occur randomly within 1 " of the centre of a galaxy this luminous at a redshift of $z<0.3$ is only $\sim 10^{-5}$. The small probability of an accidental superposition and lack of any other potential GRB host galaxies within the GRB error circle make the identification of this elliptical as the host galaxy quite secure.

The host galaxy's red colour ${ }^{20}$, spectrum and visual appearance are all consistent with a luminous elliptical galaxy and very similar to the properties of the host galaxy for the first localized short burst, GRB 050509B (ref. 1). One difference is that the host of GRB 050509B was located in a moderately rich cluster of galaxies, while the optical and X-ray observations of GRB 050724 suggest that this host elliptical is located in a lower-density region. The spectrum of the host shows no emission lines ${ }^{18}$ or evidence for recent star formation, and is consistent with a population of very old stars. This is true of most large elliptical galaxies in the present-day Universe, including the host galaxy of GRB 050509B. The elliptical hosts of these two short GRBs are very different from those for long bursts, which are typically sub-luminous, blue galaxies with strong star formation ${ }^{21}$.

Thus the properties of these two short GRB hosts suggest that the parent populations and consequently the mechanisms for short and long GRBs are different in significant ways. Their non-star-forming elliptical hosts indicate that short GRBs could not have resulted from any mechanism involving massive star core collapse $\mathrm{e}^{22}$ or recent star formation (for example, a young magnetar giant flare ${ }^{23,24}$ ). As we previously noted ${ }^{1}$, large elliptical galaxies are very advantageous sites for old, compact binary star systems, and thus good locations for neutron star-neutron star or neutron star-black hole mergers. Luminous elliptical galaxies are known to contain large populations of low-mass X-ray binaries containing neutron stars or black holes, and have large numbers of globular clusters within which compact binary stars can be formed dynamically with a much higher efficiency than in the field. Note, however, that 
mergers of compact objects are also expected to occur with a significant rate in starforming galaxies; even if such mergers are the mechanism behind all short GRBs, one would not expect them all to occur in elliptical galaxies. In fact, the second short GRB with fine localization (GRB 050709) ${ }^{2-4}$ was in a star-forming galaxy at $z=0.16$ and may be such a case.

Taking into account the host distance, we compare the energetics of short and long GRBs. The fluence in the first $3 \mathrm{~s}$ of emission is $6 \times 10^{-7} \mathrm{erg} \mathrm{cm}^{-2}$ in the 15-350 keV range, which translates roughly to a total $10 \mathrm{keV}-1 \mathrm{MeV} \gamma$-ray fluence of $\sim 10^{-6} \mathrm{erg} \mathrm{cm}^{-2}$. The fluences in the 30 to $200 \mathrm{~s}$ soft $\gamma$-ray peak and the X-ray afterglow are comparable at $7 \times 10^{-7} \mathrm{erg} \mathrm{cm}^{-2}$ and $\sim 10^{-6} \mathrm{erg} \mathrm{cm}^{-}$, respectively. These fluences are similar to those seen by BAT and other $\gamma$-ray detectors for long bursts. However, at a redshift of $z=0.285$, the total energy output of the prompt plus soft peak is $\sim 3 \times 10^{50} \mathrm{erg}$, which is significantly less than the $10^{52}-10^{54} \mathrm{erg}$ isotropic-equivalent energy output of the long GRB class ${ }^{25}$.

The early XRT lightcurve initially shows a steep decay with a slope of _2. This component is connected to the soft $\gamma$-ray component around $\sim 80 \mathrm{~s}$ identified in the extrapolated BAT lightcurve, which clearly overlaps and joins the early XRT measurements (Fig. 3). This flare-like event is followed by a very rapid decay after $\sim 100 \mathrm{~s}$ (with index <-7), interrupted by a second, less-energetic flare around 200-300 s. The curve flattens at $700 \mathrm{~s}$ and has a roughly constant decay index all the way through the Chandra points to $2 \times 10^{5} \mathrm{~s}$. A third significant flare starting at $\sim 2 \times 10^{4} \mathrm{~s}$ is superposed on this decay component, with the total energy even smaller (by a factor of $\sim 3)$ than the second one. The steep decays following all three flares are too steep to be interpreted as the afterglow emission from a forward shock, but could be consistent with the high latitude emission $(\theta>>1 / \Gamma)$ from a fireball that suddenly stops radiating or goes off in an extremely low-density medium (naked GRBs) ${ }^{26}$. This is pertinent for the late internal shock scenario as invoked to interpret the X-ray flares in long GRBs ${ }^{27}$. This interpretation requires that the central engine remains active up to at least $\sim 200 \mathrm{~s}$ (which is supported by the flaring event in the BAT-extrapolated X-ray lightcurve and the wiggles in the initial XRT lightcurve).

The current neutron star-neutron star merger models ${ }^{28,29}$ predict energy injection times much shorter than the $>200$ s time seen for GRB 050724. Black hole-neutron star mergers are more promising ${ }^{30}$, because they allow for partial disruption of the neutron star and gradual accretion as the higher angular momentum material decays through gravitational radiation, but even these models cannot extend the emission beyond a few tens of seconds. With the current Swift detection rate of one short burst per 2-3 months, 
the sample will quickly increase and will provide answers about how typical extended emission is for short burst.

Received 8 August; accepted 31 October 2005; doi:10.1038/nature04392.

1. Gehrels, N. et al. A short $\gamma$-ray burst apparently associated with an elliptical galaxy at redshift $z=0.225$. Nature 437, 851-854 (2005).

2. Villasenor, J. S. et al. Discovery of the short $\gamma$-ray burst GRB 050709. Nature 437, 855-858 (2005).

3. Fox, D. B. et al. The afterglow of GRB 050709 and the nature of short-hard $\gamma$ ray bursts. Nature 437, 845-850 (2005).

4. Covino, S. et al. Optical emission from GRB 050709: a short/hard GRB in a star-forming galaxy. Astron. Astrophys. (submitted).

5. Prochaska, J. X. et al. GRB 050724: Secure host redshift from Keck. $\boldsymbol{G C N}$ Circ. 3700 (2005).

6. Krimm, H. et al. GRB050724: Refined analysis of the Swift-BAT possible short burst. GCN Circ. 3667 (2005).

7. Berger, E. et al. A merger origin for the short GRB inferred from the afterglow and host galaxy of GRB 050724. Nature doi:10.1038/nature04238 (this issue).

8. Burrows, D. N. et al. GRB 050724: Chandra observations of the X-ray afterglow. GCN Circ. 3697 (2005).

9. Gehrels, N. et al. The Swift $\gamma$ ray burst mission. Astrophys. J. 611, 1005-1020 (2004).

10. Kouveliotou, C. et al. Identification of two classes of $\gamma$-ray bursts. Astrophys. $J$. 413, L101-L104 (1993).

11. Boer, M. et al. GRB 050709: refined spectral and temporal properties. $\boldsymbol{G C N}$ Circ. 3653 (2005).

12. Connaughton, V. BATSE observations of $\gamma$-ray burst tails. Astrophys. J. 567, 1028-1036 (2002).

13. Lazzati, D., Ramirez-Ruiz, E. \& Ghisellini, G. Possible detection of hard X-ray afterglows of short $\gamma$-ray bursts. Astron. Astrophys. 379, L39-L43 (2001).

14. Covino, S. et al. GRB 050724: a short burst detected by Swift. GCN Circ. 3665 (2005). 
15. Antonelli, L. A. et al. GRB 050724: Swift XRT refined position. GCN Circ. 3678 (2005).

16. Gal-Yam, A. et al. GRB 050724: Optical variability in a nearby galaxy. $\boldsymbol{G C N}$ Circ. 3681 (2005).

17. D'Avanzo, P. et al. GRB050724: VLT observations of the variable source. GCN Circ. 3690 (2005).

18. Bloom, J. S. et al. GRB 050724: Optical imaging/astrometry. GCN Circ. 3672 (2005).

19. Kochanek, C. S. et al. The K-band galaxy luminosity function. Astrophys. J. 560, 566-579 (2001).

20. Castro-Tirado, A. J. et al. GRB 050724: Optical and near-IR observations. GCN Circ. 3673 (2005)

21. Le Floc'h, E. et al. Are the hosts of $\gamma$-ray bursts sub-luminous and blue galaxies? Astron. Astrophys. 400, 499-510 (2003).

22. Woosley, S. E. $\gamma$-ray bursts from stellar mass accretion disks around black holes. Astrophys. J. 405, 273-277 (1993).

23. Hurley, K. et al. An exceptionally bright flare from SGR 1806-20 and the origin of short duration $\gamma$-ray bursts. Nature 434, 1098-1103 (2005).

24. Palmer, D. M. et al. A giant $\gamma$-ray flare from the magnetar SGR 1806-20. Nature 434, 1107-1109 (2005).

25. Frail, D. A. et al. Beaming in $\gamma$-ray bursts: Evidence for a standard energy reservoir. Astrophys. J. 562, L55-L58 (2001).

26. Kumar, P. \& Panaitescu, A. Afterglow emission from naked $\gamma$-ray bursts. Astrophys. J. 541, L51-L54 (2000).

27. Burrows, D. N. et al. Bright X-ray flares in $\gamma$-ray burst afterglows. Science 309, 1833-1855 (2005).

28. Rosswog, S., Ramirez-Ruiz, E. \& Davies, M. B. High-resolution calculations of merging neutron stars-III. $\gamma$-ray bursts. Mon. Not. R. Astron. Soc. 345, 1077-1090 (2003).

29. Lee, W. H., Ramirez-Ruiz, E. \& Granot, J. A compact binary merger model for the short, hard GRB 050509b. Preprint at http://arXiv.org/astro-ph0504104> (2005). 
30. Davies, M. B., Levan, A. \& King, A. The ultimate outcome of black hole-neutron star mergers. Mon. Not. R. Astron. Soc. 356, 54-58 (2005).

Acknowledgements We acknowledge support from ASI, NASA and PPARC, and acknowledge benefits from collaboration within the EU FP5 Research Training Network ' $\gamma$-Ray Bursts: An Enigma and a Tool'.

Correspondence and requests for materials should be addressed to S.D.B. (scott@,1heamail.gsfc.nasa.gov). 
Table 1 Position determinations for GRB 050724. All the positions are consistent with each other to within the errors quoted for each. See Fig 2.

\begin{tabular}{|c|c|c|c|c|c|}
\hline Observatory & RA (J2000) & Dec. (J2000) & $\begin{array}{l}\text { Error } \\
\text { circle } \\
\text { radius }\end{array}$ & Notes & Ref. \\
\hline Swift/BAT & $16 \mathrm{~h} 24 \mathrm{~min} 43 \mathrm{~s}$ & $2_{2}^{\circ} 31 \_30 \_$ & $3^{\prime}$ & $\begin{array}{l}\text { 1_ from Chandra } \\
\text { position }\end{array}$ & 6 \\
\hline Swift/XRT & $16 \mathrm{~h} 24 \mathrm{~min} 44.41 \mathrm{~s}$ & $Z_{2} 7^{\circ} 32 \_28.4$ & 6 & $\begin{array}{l}\text { Corrected } \\
\text { astrometry relative } \\
\text { to position in GCN } \\
\text { Circular } 3678\end{array}$ & 15 \\
\hline VLT & $16 \mathrm{~h} 24 \min 44.37 \mathrm{~s}$ & $27^{\circ} 32 \quad 27$ & 0.5 & & \\
\hline VLA & $16 \mathrm{~h} 24 \mathrm{~min} 44.37 \mathrm{~s}$ & $27^{\circ} 32 \_27.5$ & 0.2 & One-sigma error & 7 \\
\hline ChandralACIS & $16 \mathrm{~h} 24 \mathrm{~min} 44.36 \mathrm{~s}$ & $27^{\circ} 32 \_27.5$ & 0.5 & & 8 \\
\hline
\end{tabular}

${ }^{*} 90 \%$ confidence limit except for VLA. VLT, Very Large Telescope. VLA, Very Large Array. RA, right ascension; Dec., declination. 

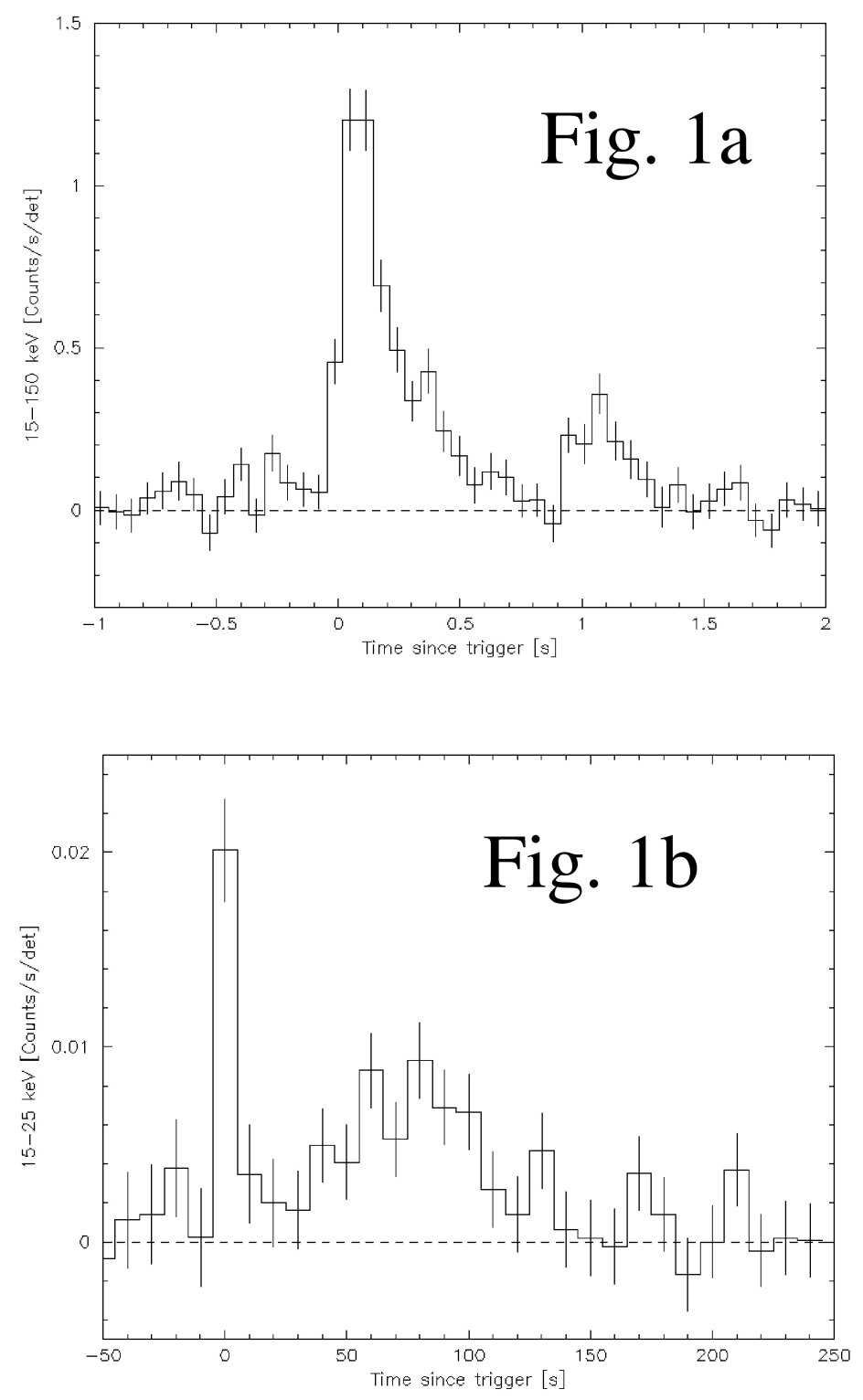

Figure 1 BAT lightcurves for GRB 050724 showing the short duration of this GRB and the long softer emission. a, The prompt emission in the $15-150 \mathrm{keV}$ energy band with a short-duration main spike of $0.25 \mathrm{~s} . T_{90}$ is $3.0 \pm 1.0 \mathrm{~s}$ ( $T_{90}$ is the time during which $90 \%$ of the GRB photons are emitted ${ }^{10}$; the fluence is $(3.9 \pm 1.0) \times 10^{-7} \mathrm{erg} \mathrm{cm}^{2}$ and the peak flux is $3.5 \pm 0.3$ photons $\mathrm{cm}^{-2} \mathrm{~s}^{-1}(15-150 \mathrm{keV}, 90 \%$ confidence level). b, Soft emission in the $15-25 \mathrm{keV}$ energy band lasting $>100 \mathrm{~s}$ (peak flux is $\sim 2 \times 10^{-9} \mathrm{erg} \mathrm{cm}^{-2} \mathrm{~s}^{-}$). The error bars in both panels are one-sigma standard deviation. The BAT energy spectrum in the prompt portion ( $T$ _ 0.03 to $T+0.29 \mathrm{~s}$; where $T$ equals BAT trigger time of 12:34:09.32 UT) is well fitted with a simple power-law model of photon index $1.38 \pm 0.13$ and normalization at $50 \mathrm{keV}$ of $0.063 \pm 0.005$ photons $\mathrm{cm}^{-}{ }^{2} \mathrm{~s}^{-1} \mathrm{keV}^{-1}(15-150 \mathrm{keV}, 90 \%$ confidence level). 


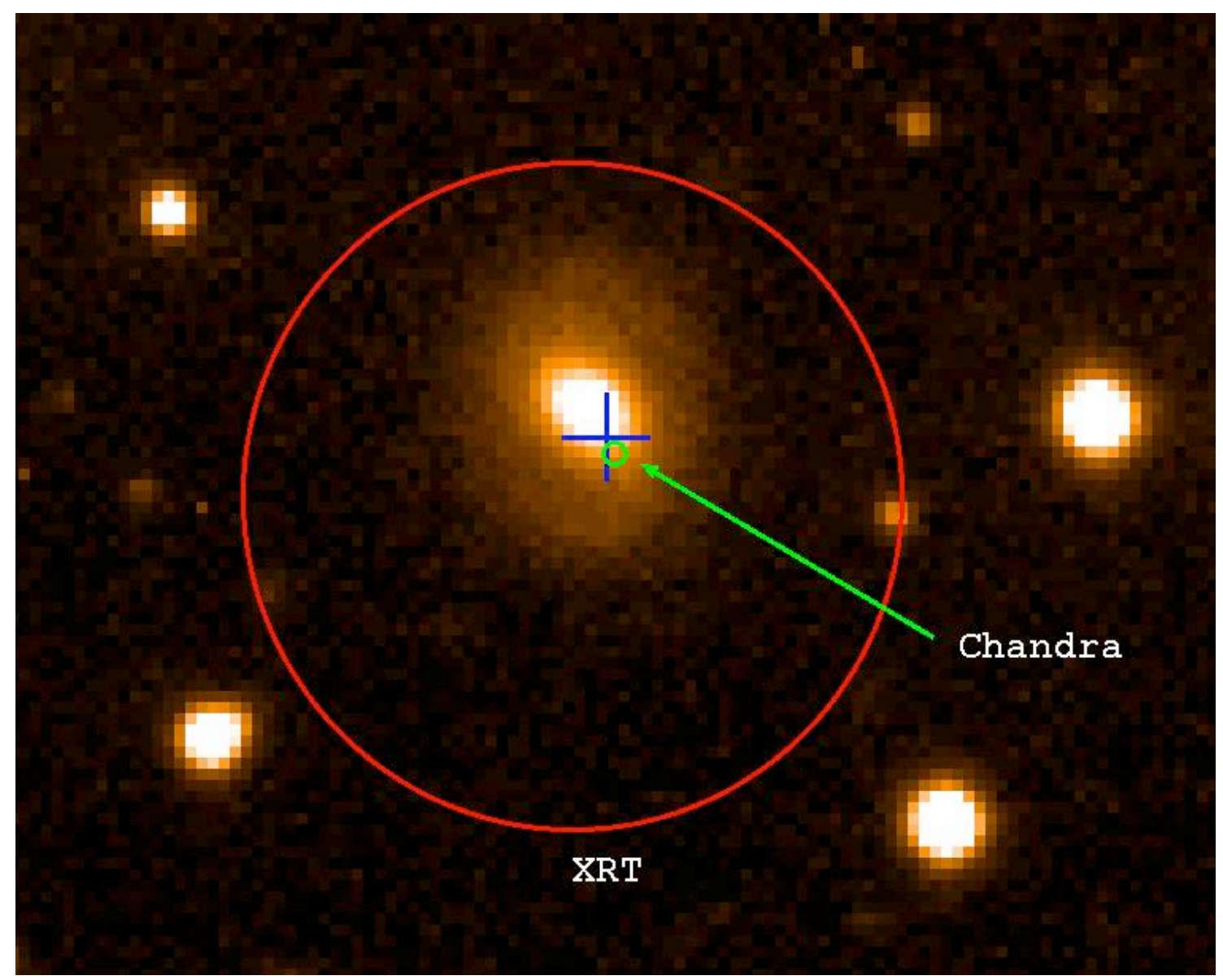

Figure 2 VLT optical image ${ }^{17}$ showing the association of GRB 050724 with the galaxy. The blue cross is the position of the optical transient ${ }^{16,17}$. The XRT (red circle) and Chandra (green circle) burst positions are superimposed on a bright red galaxy at redshift $z=0.258$ (ref. 5), implying a low-redshift elliptical galaxy as the host. The XRT position has been further revised from the position of ref. 15 by astrometric comparison with objects in the field. The projected offset from the centre of the galaxy corresponds to $\sim 4 \mathrm{kpc}$ assuming the standard cosmology with $H_{0}=71 \mathrm{~km} \mathrm{~s}^{-}{ }^{1} \mathrm{Mpc}^{-}{ }^{1}$ and $\left(\Omega_{\mathrm{M}}\right.$, $\left.\Omega_{\Lambda}\right)=(0.27,0.73)$. 


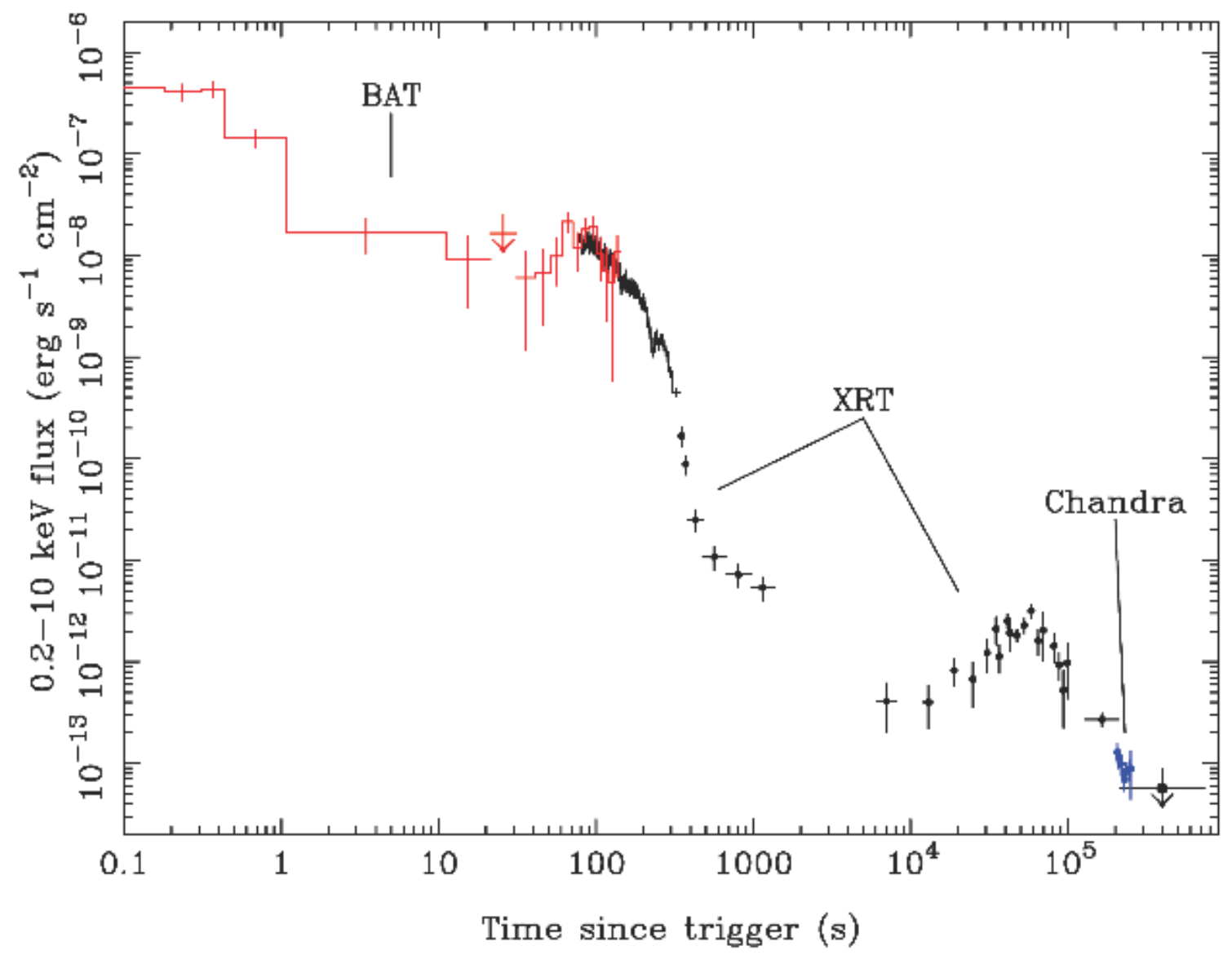

Figure 3 The smooth transition of the GRB phase into the X-ray phase. This shows the total X-ray afterglow lightcurve for GRB 050724 by combining data from BAT, XRT and Chandra. The prompt hard phase detected by BAT decays into the soft $\gamma$ emission, and is followed by flaring $(\sim T+80 \mathrm{~s})$ and then gradual fall-off into the X-ray afterglow measured by XRT. The BAT points are binned with variable time intervals such that the signal-to-noise ratio is $>5$ and $\Delta T<10 \mathrm{~s}$. Using the Band model to simultaneously fit the BAT spectra in the $15-150 \mathrm{keV}$ band in the $T+(50-150) \mathrm{s}$ time interval with the XRT spectra in the $0.2-10 \mathrm{keV}$ band in the $T+(79-150) \mathrm{s}$ interval (yielding $E_{\text {break }}=30 \mathrm{keV}$ ), the BAT flux points $(15-25 \mathrm{keV}$ band) were scaled down into the X-ray band (unabsorbed $0.2-10 \mathrm{keV}$ ). The Band model was required because power-law fits to the BAT and XRT separately yielded $\Gamma$ values of 2.5 and 1.7, respectively: that is, there was a spectral break between the two instruments. The XRT fluxes are unabsorbed values derived using best-fit XRT photon indices of $\Gamma=1.9$ (for the 'window timing' mode data before $341 \mathrm{~s}$ ) and $\Gamma=1.5$ (for the 'photon counting' mode data after $342.9 \mathrm{~s}$ ) and using the best-fit absorbing column density of $N_{\mathrm{H}}=5.9 \times 10^{21} \mathrm{~cm}^{-}{ }^{2}$. All error bars are one-sigma standard deviation. The Chandra points are unabsorbed fluxes using the XRT-measured value for $N_{\mathrm{H}}$ and the Chandra-measured best-fit photon index of $\Gamma=1.8$. 